\title{
Antibody Fc effector functions and IgG3 associate with decreased HIV-1 risk
}

\author{
Scott D. Neidich, ${ }^{1}$ Youyi Fong, ${ }^{2,3,4}$ Shuying S. Li, ${ }^{2,3}$ Daniel E. Geraghty, ${ }^{5}$ Brian D. Williamson, ${ }^{4}$ William Chad Young, ${ }^{2}$ \\ Derrick Goodman, ${ }^{1}$ Kelly E. Seaton, ${ }^{1}$ Xiaoying Shen, ${ }^{1}$ Sheetal Sawant, ${ }^{1}$ Lu Zhang, ${ }^{1}$ Allan C. deCamp, ${ }^{2}$ Bryan S. Blette, ${ }^{6}$ \\ Mengshu Shao, ${ }^{2}$ Nicole L. Yates, ${ }^{1}$ Frederick Feely, ${ }^{1}$ Chul-Woo Pyo, ${ }^{3}$ Guido Ferrari, ${ }^{1,7,8}$ HVTN 505 Team, ${ }^{9}$ Ian Frank, ${ }^{10}$ \\ Shelly T. Karuna, ${ }^{3}$ Edith M. Swann, ${ }^{11}$ John R. Mascola, ${ }^{12}$ Barney S. Graham, ${ }^{12}$ Scott M. Hammer, ${ }^{13}$ Magdalena E. Sobieszczyk, ${ }^{13}$ \\ Lawrence Corey, ${ }^{3}$ Holly E. Janes, ${ }^{2,3,4}$ M. Juliana McElrath, ${ }^{3}$ Raphael Gottardo,,$^{2,3}$ Peter B. Gilbert, ${ }^{2,3,4}$ and Georgia D. Tomaras ${ }^{1,7,8,14}$ \\ 'Duke Human Vaccine Institute, Duke University, Durham, North Carolina, USA. ${ }^{2}$ Statistical Center for HIV/AIDS Research and Prevention, ${ }^{3}$ Vaccine and Infectious Disease Division, Fred Hutchinson Cancer \\ Research Center, Seattle, Washington, USA. ${ }^{4}$ Department of Biostatistics, University of Washington, Seattle, Washington, USA. ${ }^{5}$ Clinical Research Division, Fred Hutchinson Cancer Research Center, Seattle, \\ Washington, USA. ${ }^{6}$ Department of Biostatistics, University of North Carolina Gillings School of Global Public Health, Chapel Hill, North Carolina, USA. 'Department of Surgery and ${ }^{8}$ Department of Molecular \\ Genetics and Microbiology, Duke University, Durham, North Carolina, USA. ${ }^{9}$ The HVTN 505 Team is detailed in the Supplemental Acknowledgments. ${ }^{10}$ Division of Infectious Diseases, Perelman School \\ of Medicine, University of Pennsylvania, Philadelphia Pennsylvania, USA. "Division of AIDS and ${ }^{12}$ Vaccine Research Center, National Institute of Allergy and Infectious Diseases (NIAID), NIH, Bethesda, \\ Maryland, USA. ${ }^{13}$ Division of Infectious Diseases, Department of Medicine, Columbia University, New York, New York, USA. ${ }^{14}$ Department of Immunology, Duke University, Durham, North Carolina, USA.
}

\begin{abstract}
HVTN 505 is a preventative vaccine efficacy trial testing DNA followed by recombinant adenovirus serotype 5 (rAd5) in circumcised, Ad5-seronegative men and transgendered persons who have sex with men in the United States. Identified immune correlates of lower HIV-1 risk and a virus sieve analysis revealed that, despite lacking overall efficacy, vaccine-elicited responses exerted pressure on infecting HIV-1 viruses. To interrogate the mechanism of the antibody correlate of HIV-1 risk, we examined antigen-specific antibody recruitment of $\mathrm{Fc} \gamma$ receptors ( $\mathrm{Fc} \gamma \mathrm{Rs}$ ), antibody-dependent cellular phagocytosis (ADCP), and the role of anti-envelope (anti-Env) IgC3. In a prespecified immune correlates analysis, antibody-dependent monocyte phagocytosis and antibody binding to FcyRIla correlated with decreased HIV-1 risk. Follow-up analyses revealed that anti-Env IgG3 breadth correlated with reduced HIV-1 risk, anti-Env IgA negatively modified infection risk by Fc effector functions, and that vaccine recipients with a specific FcyRIla single-nucleotide polymorphism locus had a stronger correlation with decreased HIV-1 risk when ADCP, Env-Fc $\gamma$ RIla, and IgC3 binding were high. Additionally, Fc $\gamma$ RIla engagement correlated with decreased viral load setpoint in vaccine recipients who acquired HIV-1. These data support a role for vaccine-elicited anti-HIV-1 Env IgC3, antibody engagement of FcRs, and phagocytosis as potential mechanisms for HIV-1 prevention.
\end{abstract}

\section{Introduction}

An efficacious vaccine that provides durable immunity remains a key priority in the fight against HIV-1. One strategy to identify an efficacious vaccine regimen is the identification of immune correlates of risk from human efficacy trials and nonhuman primate studies $(1,2)$. The most recently concluded HIV-1 efficacy trial, HIV Vaccine Trials Network (HVTN) 505, tested a DNA/ recombinant adenovirus serotype 5 (rAd5) vaccine regimen in

\section{Related Commentary: p. 4580}

Authorship note: The first author position is shared by SDN, YF, and SSL. PBG and CDT are co-senior authors

Conflict of interest: PBG is the recipient of a contract from Sanofi Pasteur. GDT is a recipient of a research subcontract from CSK. PBG has served as an unpaid consultant at advisory meetings to Sanofi Pasteur and has received support for travel to meetings from Sanofi Pasteur. GDT has patent applications for HIV immunogens (nos. 14/130338, 201180021030.5, 13/262706, 15/195545, and 15/025,031) and HIV incidence testing (16/315,688 HIV-1). SDN's spouse receives a salary from Nuventra Pharma Sciences. Copyright: 다 2019, American Society for Clinical Investigation.

Submitted: November 21, 2018; Accepted: August 7, 2019; Published: October 7, 2019. Reference information: / Clin Invest. 2019;129(11):4838-4849. https://doi.org/10.1172/JCl126391.
US-based men and transgender persons who have sex with men; the trial reported no overall vaccine efficacy (3). This vaccine regimen did influence acquisition of infection as reported in a virus sieve analysis by deCamp et al. that revealed that HIV-1 envelope (Env) gp120 sequences in vaccinees who became infected were significantly more distant from the vaccine subtype B insert than infections in the placebo group (4). Moreover, a cellular immune correlate of risk found that HIV-1 Env CD8 ${ }^{+} \mathrm{T}$ cell polyfunctionality inversely correlated with HIV-1 acquisition (5). Among vaccinees with low $\mathrm{CD}^{+} \mathrm{T}$ cell polyfunctionality, anti-Env IgG correlated with reduced risk for HIV-1 acquisition (6), signifying that both vaccine-elicited $\mathrm{CD} 8^{+} \mathrm{T}$ cells and antibodies could be useful in efficacious vaccine design. Possible mechanisms for antibody-mediated prevention include a variety of antibody effector functions (reviewed in ref. 7). Given the high response rate for antibody-dependent cellular phagocytosis (ADCP) (4) elicited by this vaccine regimen, and the association of antibody effector function in rhesus macaque challenge studies (8-12), we hypothesized that ADCP and antigen-specific recruitment of Fc $\gamma$ receptors (Fc $\gamma$ Rs) were associated with reduced HIV-1 risk in HVTN 505.

Vaccine-elicited antibodies can recruit and activate innate immune effector cells, which express Fc $\gamma$ Rs that differentially 
A

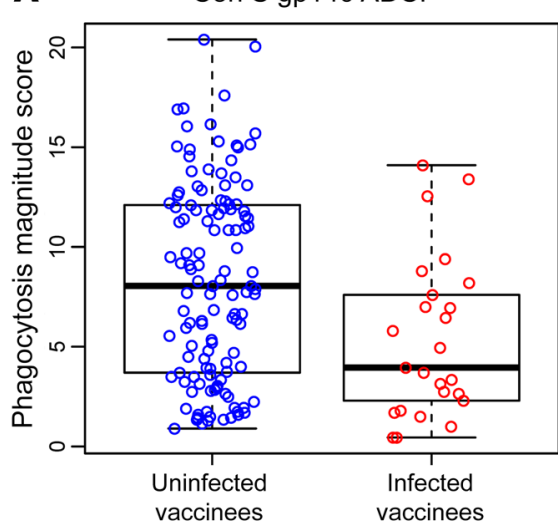

B

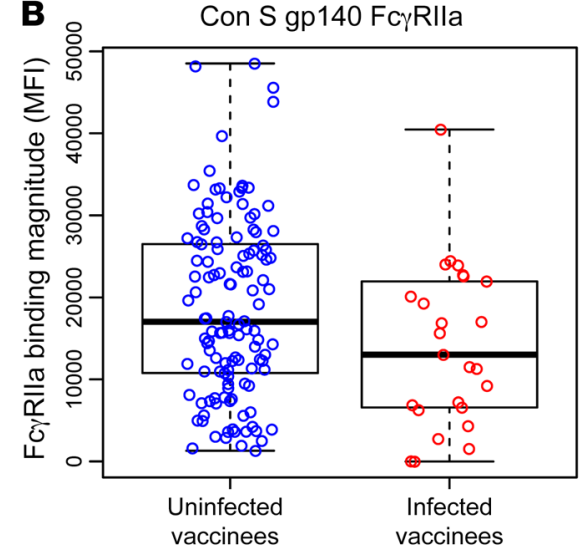

C

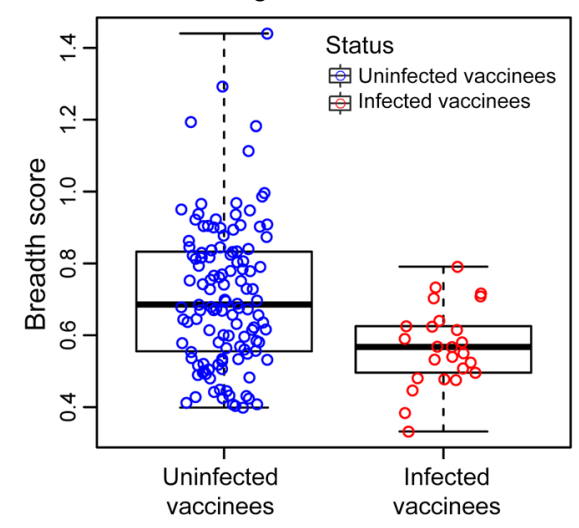

Figure 1. ADCP, FcyRIla, and IgG3 significantly correlate with decreased HIV-1 risk. HIV-1 Env Con S gp140 ADCP magnitude (score) (A), FcyRIla binding $\mathrm{MFI}(\mathrm{B})$, and IgG3 breadth score (C) by infected/uninfected outcome. ADCP (OR $=0.47, P=0.001)$, Fc $\gamma \mathrm{RIIa}(\mathrm{OR}=0.48, P<0.001)$, and Env IgG3 breadth $(\mathrm{OR}=0.326, P<0.001)$ were inversely correlated with HIV-1 acquisition risk. Box-and-whisker plots show the median line and interquartile ranges. $n=125$ uninfected, 25 infected participants.

engage antibody Fc regions (7). Fc $\gamma \mathrm{R}$ engagement links the specificity of the humoral immune response to the effector functions provided by cells of the innate immune system, as exemplified by antibody-dependent cell-mediated cytotoxicity (ADCC) (13) and ADCP (14). Although these potent antiviral activities are regulated by an interaction of both activating and inhibitory Fc $\gamma$ Rs, Fc $\gamma$ RIIIa and -IIa are most closely associated with ADCC and ADCP, respectively $(7,13,14)$. Although signaling pathways triggered by Fc $\gamma$ Rs share downstream targets, ADCP can be blunted through selective blockade of Fc $\gamma \mathrm{RI}$ and -II, and this blunting is enhanced with blocking of Fc $\gamma$ RIII (15). ADCC is mediated predominantly by Fc $\gamma$ RIIIa; however, it can be enhanced by Fc $\gamma$ RII signaling (16). Interestingly, the expression of Fc $\gamma$ RII and Fc $\gamma$ RIIIa is also diminished on innate immune cells isolated from HIV-1-infected individuals (15), and Fc-mediated functions were associated with the 31\% efficacy observed in the Thai RV144 vaccine trial $(17,18)$. Insights into the antibody mechanisms of HIV-1 correlates of risk from recent HIV-1 vaccine trials will enable advances in vaccine immunogen design toward a globally efficacious HIV-1 vaccine.

\section{Results}

HIV-1 Env IgG-mediated phagocytosis and Fc $\gamma$ RIIa engagement correlates with decreased HIV-1 risk in a human HIV-1 vaccine efficacy trial. Given the high response rate and broad dynamic range in the magnitude of ADCP in a pilot study of HVTN 505 immunogenicity (4), we designed an antibody function immune correlates analysis to test the prespecified hypothesis that anti-HIV-1 Env IgG ADCP and engagement of Fc $\gamma$ RIIa correlates with decreased HIV-1 acquisition risk. The response rate and magnitude of these antibody functions were determined in a blinded case control study of 125 uninfected and 25 infected vaccinees at 4 weeks after the final vaccination (month 7) (infection cases were diagnosed between month 7 and month 24). Env IgG ADCP was elicited in $88.0 \%$ (81.8\%-92.3\% $\mathrm{CI}$ ) of vaccine recipients, with a median ADCP score of 8.1 (range 0.9-20.4) for uninfected vaccinees and a median ADCP score of 4.0 (range 0.45-14.1) for infected vaccinees (Figure 1, A and B). The response rate for HIV-1 Env Con S gp140 Fc $\gamma$ RIIa was 98.7\% (Supplemental Figure 1 and Supplemental Table 1; supplemental material available online with this article; https://oi.org/10.1172/ JCI126391DS1). In a regression analysis controlling for age, race, behavior risk, and body mass index (BMI), the primary hypothesis outcomes of IgG ADCP and Fc $\gamma$ RIIa responses to HIV-1 Env glycoprotein Con S gp140 were both inversely correlated with HIV-1 acquisition risk (Table 1). Notably, a virus sieve signature previously reported for this trial (4) was detected using the Env Con S gp140 sequence (Supplemental Figure 3). To test whether these antibody functions were independent correlates of HIV-1 risk, ADCP and Fc $\gamma$ RIIa were assessed as univariate variables while controlling for $\mathrm{CD}^{+}$and $\mathrm{CD}^{+} \mathrm{T}$ cell polyfunctionality score (PFS). With this adjustment, Fc $\gamma$ RIIa and ADCP remained significantly correlated with reduced HIV-1 acquisition risk and there was no interaction with CD $8^{+} \mathrm{T}$ cell polyfunctionality (Figure $1, \mathrm{~A}$ and $\mathrm{B}$, and Table 1 ).

$H I V-1$ Env IgG3 correlates with decreased HIV-1 risk in a human $H I V-1$ vaccine efficacy trial. Since we and others previously report-

Table 1. Primary hypothesis analysis of risk for HIV-1 acquisition among HVTN 505 vaccinees

\begin{tabular}{|c|c|c|c|c|c|c|}
\hline \multirow[b]{2}{*}{ Analysis } & \multicolumn{3}{|c|}{$A D C P$} & \multicolumn{3}{|c|}{ FcyRlla H131-Con S gp140 } \\
\hline & OR & $P$ value & $\underline{\underline{Q} \text { value }}$ & OR & $P$ value & $\underline{Q}$ value \\
\hline Univariate $^{B}$ & 0.47 & 0.001 & 0.001 & 0.48 & $<0.001$ & $<0.001$ \\
\hline Univariate w/ cellular adjustment ${ }^{\complement}$ & 0.59 & 0.026 & 0.026 & 0.63 & 0.010 & 0.021 \\
\hline CD8 interaction $^{0}$ & 1.24 & 0.448 & 0.672 & 1.34 & 0.280 & 0.672 \\
\hline
\end{tabular}

${ }^{A}$ Log-transformed (In[MFI]) values for FcyRIla H131-Con S gp140. ${ }^{B}$ Univariate analyses to assess if the variable (ADCP or Fc $\gamma$ RIla H131-Con S gp140) was associated with HIV-1 acquisition risk. ${ }^{\circ}$ The addition of cellular adjustment adjusts for previously published CD4 and CD8 polyfunctionality scores. ${ }^{\circ} \mathrm{CD} 8$ interaction assesses for an interaction between CD8 polyfunctionality score and the variable tested. 
Table 2. IgG3 analysis of HIV-1 acquisition among HVTN 505 participants

\begin{tabular}{|c|c|c|c|c|c|c|c|c|c|}
\hline & \multicolumn{3}{|c|}{ Univariate } & \multicolumn{3}{|c|}{ Univariate w/ cellular adjustment } & \multicolumn{3}{|c|}{ CD8 interaction } \\
\hline & $\mathrm{OR}$ & $P$ value & $\underline{\underline{Q} \text { value }}$ & $\mathrm{OR}$ & $P$ value & $\underline{\underline{O} \text { value }}$ & OR & $P$ value & $\underline{Q}$ value \\
\hline A Con gp140 & 0.330 & $<0.001$ & $<0.001$ & 0.369 & $<0.001$ & 0.001 & 1.281 & 0.316 & 0.536 \\
\hline B Con gp140 & 0.347 & $<0.001$ & $<0.001$ & 0.433 & 0.001 & 0.003 & 1.166 & 0.521 & 0.681 \\
\hline Con S gp140 & 0.466 & 0.001 & 0.002 & 0.531 & 0.004 & 0.012 & 1.064 & 0.786 & 0.835 \\
\hline C Con gp140 & 0.391 & $<0.001$ & 0.001 & 0.402 & 0.001 & 0.003 & 1.292 & 0.315 & 0.536 \\
\hline VRC A gp140 & 0.468 & $<0.001$ & 0.001 & 0.517 & 0.001 & 0.005 & 1.458 & 0.190 & 0.536 \\
\hline VRC B gp140 & 0.341 & $<0.001$ & $<0.001$ & 0.368 & $<0.001$ & 0.001 & 1.324 & 0.273 & 0.536 \\
\hline VRC C gp140 & 0.566 & 0.007 & 0.016 & 0.573 & 0.017 & 0.040 & 1.091 & 0.740 & 0.835 \\
\hline Con 6 gp120 & 0.463 & 0.018 & 0.039 & 0.480 & 0.042 & 0.088 & 1.204 & 0.573 & 0.695 \\
\hline VRC A gp70 V1V2 & 0.981 & 0.921 & 0.921 & 0.998 & 0.993 & 0.993 & 1.356 & 0.176 & 0.536 \\
\hline p24 & 0.674 & 0.062 & 0.105 & 0.662 & 0.082 & 0.139 & 1.247 & 0.472 & 0.669 \\
\hline gp41 & 0.649 & 0.047 & 0.088 & 0.826 & 0.382 & 0.464 & 1.433 & 0.164 & 0.536 \\
\hline
\end{tabular}

Association of V1V2 IgG3 with HIV-1 risk is shown only for the measurement $>20 \%$ response rate. ic plasma IgA correlated with increased HIV-1 risk (i.e., associated with decreased vaccine efficacy) in the RV144 clinical trial and reported that certain specificities may inhibit antibody Fc effector functions $(17,18)$. To determine the role of HIV-1 Env IgA in this study, we examined whether vaccine-elicited IgA responses modified the correlation of antibody function with HIV-1 acquisition risk. In vaccinees with low-to-undetectable plasma HIV-1 Env gp140-specific IgA responses, the association of ADCP and Fc $\gamma$ RIIa binding with risk of $\mathrm{HIV}-1$ ed that HIV-1-specific IgG3 antibodies have improved ADCP activity over IgG1 (19) and that HIV-specific IgG3 correlated with reduced HIV-1 acquisition, (20), we next examined the role of IgG3 as an exploratory hypothesis in this study. Vaccine-matched gp140 IgG3 response rates were high, ranging from 55\% to $90 \%$ (Supplemental Figure 2). Notably, Env IgG3 breadth (odds ratio $[\mathrm{OR}]=0.326, P<0.001 ; \mathrm{OR}=0.365, P$ value $<0.001$ with cellular adjustment), as well as individual Env IgG3 measurements significantly correlated with reduced risk of HIV-1 acquisition (Figure $1 \mathrm{C}$ and Table 2). V1V2 IgG3 response rates were very low (<20\%) (Supplemental Figure 2) and were not included in the test for association with HIV-1 acquisition.

IgA modifies the association of antibody-mediated phagocytosis and Fc $\gamma$ RIIa function with HIV-1 risk. We previously demonstrated that HIV-1 Env-specif-

Figure 2. Envelope-specific IgA modifies the association of ADCP and Fc $\gamma$ RIla with HIV-1 risk. HIV-1 envelope Con S gp140 ADCP magnitude (score) (A) and Fc $\gamma$ Rlla binding MFI (B) by infected/ uninfected outcome, stratified by Env IgA positivity. The associations between ADCP/Fc $\gamma$ RIla and HIV-1 acquisition risk are stronger among vaccinees without detectable Env IgA than those with detectable Env IgA (ADCP OR = 0.16, $P=0.006$; $\mathrm{F} c \gamma \mathrm{R}$ Ila $\mathrm{OR}=0.37, P<0.001)$. The interaction is significant for $\operatorname{ADCP}$ (ratio of ORs $=4.3, P=$ 0.039 ) but not for Fc $\gamma$ Rlla (ratio of ORs $=1.5$, $P=0.43)$. ADCP and FcyRlla did impact risk in IgA-positive participants $\left(\mathrm{ADCP} \lg \mathrm{A}^{+} \mathrm{OR}=\right.$ $0.68, P=0.011$; Fc $\gamma$ RIla IgA ${ }^{+}$OR $=0.56, P<$ 0.001 ). Statistics are summarized in Table 3. $P$ values were calculated using the Wald test. Box-and-whisker plots show the median line and interquartile ranges. $n=125$, uninfected, 25 infected participants.
A

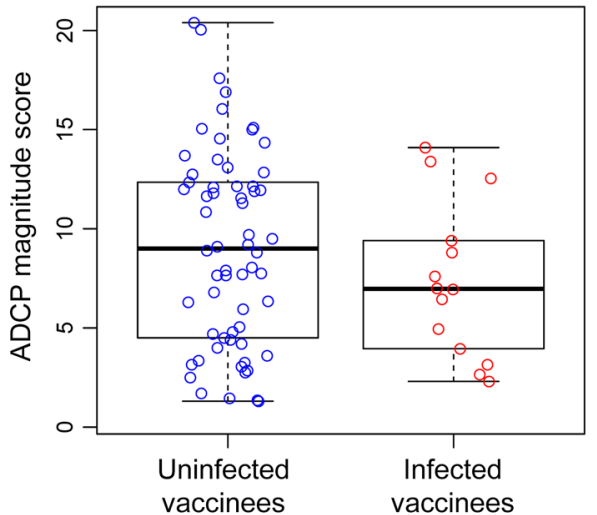

B

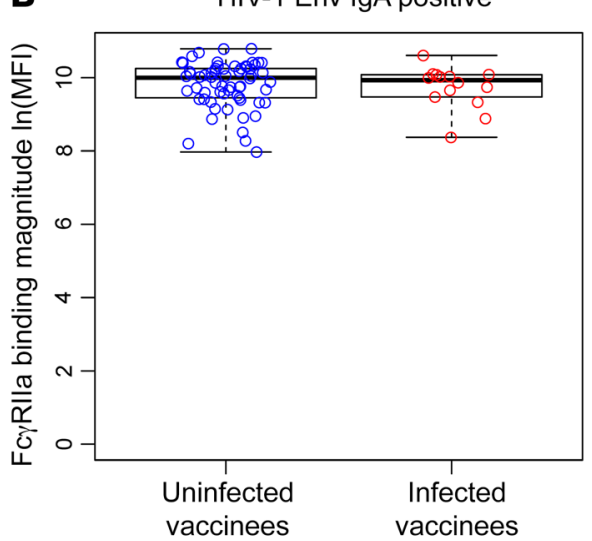

HIV-1 Env IgA negative

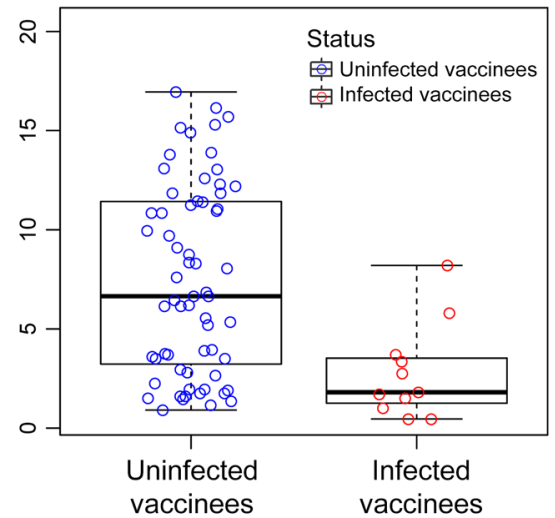

HIV-1 Env IgA negative

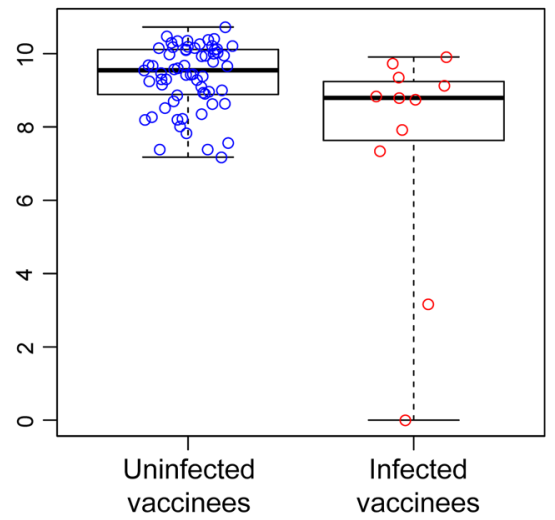


Table 3. Assessment of risk stratified by serum IgA positivity

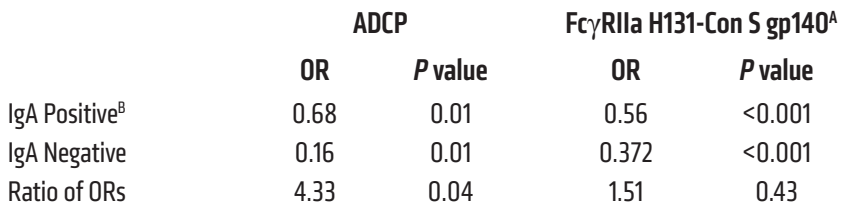

${ }^{A}$ Log-transformed (In[MFI]) values for FcyRIla H131-Con S gp140. ${ }^{\mathrm{B}} \mathrm{gA}$ positivity is defined as 3 -fold increase over baseline and above the 95th percentile of control sera.

Secondary and exploratory analyses. In addition to the primary hypothesis, we tested an array of HIV-1-specific Env proteins for Fc $\gamma$ RIIa and Fc $\gamma$ RIIIa engagement. These variables were examined as univariate parameters in a predefined secondary-tier (Table 4) or exploratory-tier (Table 5) analysis with significance defined as a $P$ value of less than 0.05 and $Q$ value of less than 0.2 , to adjust for multiple comparisons, in part prioritized based on outcomes from the binding antibody correlates analysis (6). To understand whether there was an interplay of cellular and humoral responses, we next examined antibody interactions with polyfunctional HIV-1-specific $\mathrm{CD}^{+} \mathrm{T}$ cells and $\mathrm{CD} 8^{+} \mathrm{T}$ cells. Although several of the secondaryand exploratory-tier analytes met significance cutoff for univariate analyses $(P<0.05, Q<0.2)$, none maintained significance following adjustment for cellular responses and corrections for multiple comparisons, suggesting that any effect driven by binding against these antigens was predominantly associated with one of the other significant correlates. It is worth noting that all measurements for Fc $\gamma \mathrm{R}$ binding were unidirectional; no significant OR for Fc $\gamma$ RIIa or Fc $\gamma$ RIIIa exceeded 1, meaning none were associated with increased HIV-1 acquisition. Similarly to the primary-tier analysis, there were no significant interactions of these antibody-FcR interactions with measurements of $\mathrm{CD}^{+} \mathrm{T}$ cell functions. Since obesity was implicated in increasing infection risk in the context of influenza vaccination (21) and was reported to negatively impact seroprotection in the context of hepatitis B vaccination $(22,23)$, we also explored the role of $\operatorname{BMI}\left(\geq 30 \mathrm{~kg} / \mathrm{m}^{2}\right)$ in modulating the correlation of antibody function and HIV-1 acquisition. There was no significant effect of BMI $(P>$ 0.05 by Wilcoxon's rank-sum

test and $Q>0.2)$.

Systems immunology analysis: multiple immune responses among vaccinees contribute to potential protection. To determine if a complex relationship existed between all known immune measures established to influence HIV-1 acquisition risk in HVTN 505, a principal component analysis (PCA) and SuperLearner approaches were applied (Figure 3). Variables included in the PCA analysis (Figure 3A and Supplemental Table 3), were immune responses associated with HIV-1 acquisition risk in this study or prior studies: Env $\mathrm{CD}^{+} \mathrm{T}$ cell PFS (5), Env-specific IgA (5), gp41- and Env-specific IgG (5), V2-specific IgG (5), ADCP, Env IgG3, and binding to Fc $\gamma$ RIIa. The first 2 principal components together accounted for $63 \%$ of the total variance. The first principal component (PC1) included high Env- and gp41-specific IgG, ADCP, IgG3, and binding to Fc $\gamma$ RIIa, while PC2 included high $\mathrm{CD}^{+} \mathrm{T}$ cell polyfunctionality, high Env-specific IgG3, and low Env-specific IgA (Supplemental Table 2). Higher PC1 was inversely correlated with HIV-1 risk $(\mathrm{OR}=0.366, P<0.001)$, and higher PC2 was also inversely correlated with HIV-1 risk $(\mathrm{OR}=0.409, P \leq$ 0.001 ) in a multivariate analysis (Figure $3 \mathrm{~A}$ ). IgG3 contributed to both PC1 and PC2 (Supplemental Table 1). We also tested interaction between PC1 and PC2 in a regression model and found evidence for a potential synergistic effect between the two (ratio of ORs $=1.653, P=0.040$ ). To further test whether HIV-1 infection risk in this cohort could be predicted from multiple humoral and cellular immune responses together, we utilized a SuperLearner (24) approach to predict HIV-1 infection in vaccinees from all measured immune responses (i.e., $\mathrm{CD}^{+}{ }^{+} \mathrm{T}$ cells, $\mathrm{CD} 8^{+} \mathrm{T}$ cells, IgG, IgA, IgG3, ADCP, Fc $\gamma$ RIIa, and Fc $\gamma$ RIIIa) (not conditioning on being univariate correlates) and baseline variables (age, BMI, behavioral risk score). In this model, where a cross-validated area under the curve (CV-AUC) of 1.00 would be perfect prediction and 0.50 would be random prediction, the overall prediction based on baseline variables and all immune measurements CV-AUC was 0.748 (95\% CI 0.652-0.844) (Figure 3B). By differentiating the importance of different classes in variables (including functional antibody measures such as FcR binding and ADCP), IgG binding, IgA binding, IgG3 binding, $\mathrm{T}$ cell measures, and permutations combining these measures), it was observed that (i) IgG and IgA improved prediction compared with baseline variables alone; (ii) adding IgG3 variables improved prediction modeling more than (i); (iii) adding $\mathrm{T}$ cell variables enhanced prediction better than (i, ii); and (iv) adding functional antibodies to $\mathrm{T}$ cells improved prediction. The individual cellular immune measurement that best predicted HIV-1 acquisition was polyfunctional $\mathrm{CD} 8^{+} \mathrm{T}$ cells and the individual humoral immune measurement that best predicted HIV-1 acquisition was Env IgG3 breadth. Finally (v), combining $\mathrm{T}$ cell and antibody variables further enhanced prediction more
Table 4. Secondary-tier analysis of HIV-1 acquisition among HVTN 505 vaccinees

\begin{tabular}{|c|c|c|c|c|c|c|c|c|c|}
\hline & \multicolumn{3}{|c|}{ Univariate } & \multicolumn{3}{|c|}{ Univariate w/ cellular adjustment } & \multicolumn{3}{|c|}{ CD8 interaction } \\
\hline & OR & $P$ value & $\underline{Q}$ value & OR & $P$ value & $\underline{\underline{Q} \text { value }}$ & OR & $P$ value & $\underline{\underline{Q} \text { value }}$ \\
\hline FcyRlla AE.A244 V1V2 Tags & 1.123 & 0.600 & 0.600 & 1.239 & 0.403 & 0.453 & 1.267 & 0.407 & 0.454 \\
\hline FcyRlla C.Con gp140 & 0.592 & 0.011 & 0.025 & 0.779 & 0.209 & 0.376 & 1.395 & 0.277 & 0.356 \\
\hline FcyRlla VRC A gp140 & 0.598 & 0.006 & 0.019 & 0.724 & 0.053 & 0.268 & 1.619 & 0.075 & 0.299 \\
\hline FcyRlla VRC C gp140 & 0.646 & 0.017 & 0.031 & 0.824 & 0.341 & 0.453 & 1.754 & 0.028 & 0.256 \\
\hline FcyRIIla Con S gp140 & 0.545 & 0.006 & 0.019 & 0.687 & 0.059 & 0.268 & 1.399 & 0.211 & 0.356 \\
\hline FcyRllla AE.A244 V1V2 Tags & 1.283 & 0.226 & 0.254 & 1.327 & 0.371 & 0.453 & 1.229 & 0.454 & 0.454 \\
\hline FcyRIIlla C.Con gp140 & 0.526 & 0.004 & 0.019 & 0.686 & 0.099 & 0.297 & 1.399 & 0.269 & 0.356 \\
\hline FcyRllla VRC A gp140 & 0.634 & 0.056 & 0.084 & 0.767 & 0.144 & 0.323 & 1.531 & 0.100 & 0.299 \\
\hline FcyRIIlla VRC C gp140 & 0.685 & 0.080 & 0.103 & 0.870 & 0.533 & 0.533 & 1.526 & 0.135 & 0.304 \\
\hline
\end{tabular}

Bold text indicates significant $P$ value $(<0.05)$, with $\underline{Q}<0.20$. 
Table 5. Exploratory-tier analysis of HIV-1 acquisition among HVTN 505 participants

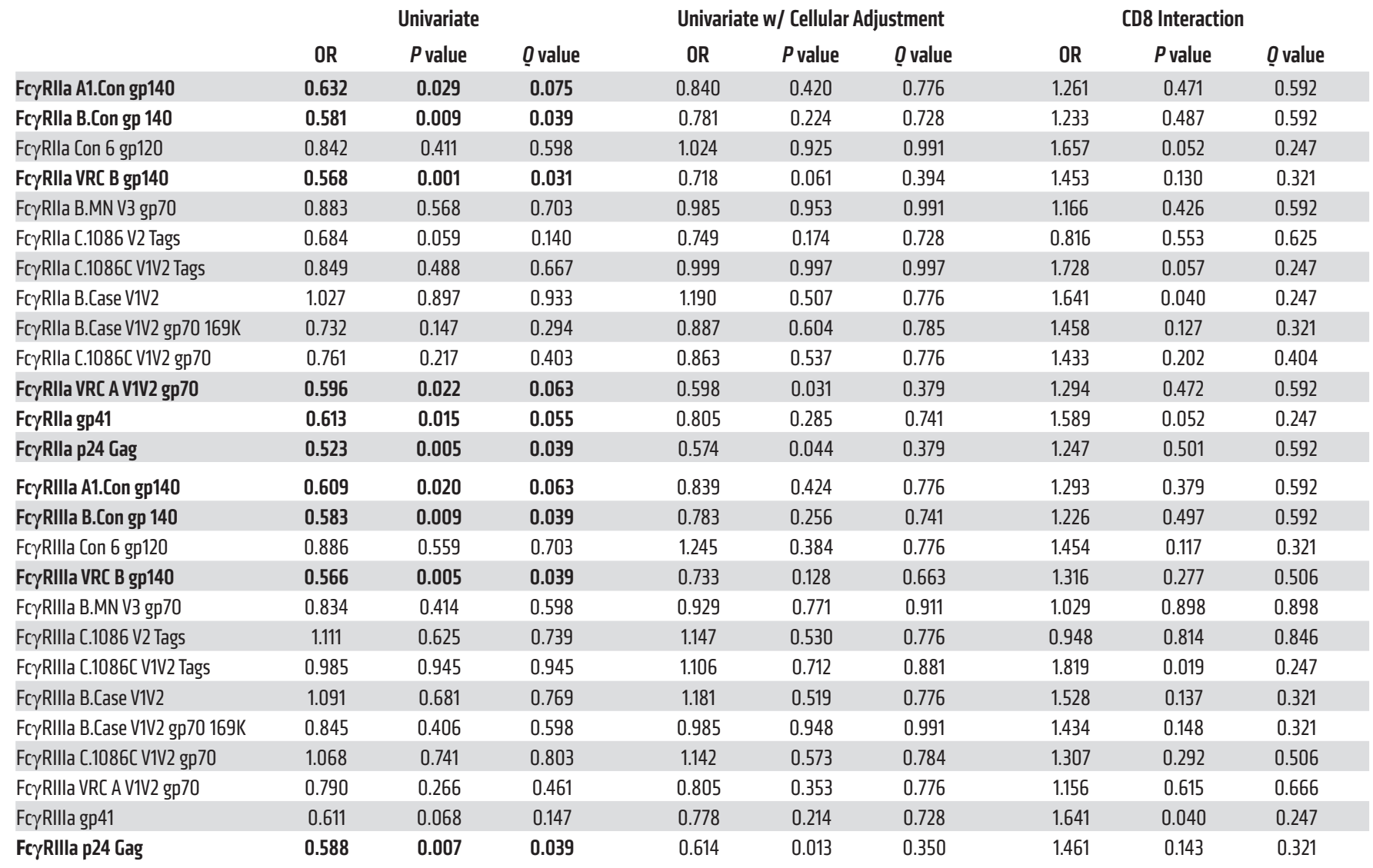

Bold text indicates significant $P$ value $(<0.05)$, with $\underline{0}<0.20$.

potently than (i, ii, iii, or iv) alone, signifying that each of these immune measures is predictive of HIV-1 risk in this model.

To further examine the relationship of vaccine-elicited immunity across humoral and cellular responses, we modeled vaccine immune responses at different levels that most strongly correlated with decreased HIV-1 risk (Figure 4). The antibody function of ADCP and Env IgG3 at month 7, i.e., 4 weeks after the last vaccination, when cut at above and below the median response magnitude stratified vaccinees into vaccine efficacy (VE) greater than zero and VE near zero at about the same strength as the cut by Env $\mathrm{CD}^{+} \mathrm{T}$ cell PFS (ref. 5 and Figure $4, \mathrm{~A}-\mathrm{C}$ ). VE is defined as 1 minus the relative risk (vaccine vs. placebo) of infection by month 24 . In particular, based on a causal inference method that estimates VE in subgroups of vaccine recipients defined by an immune response measurement if assigned vaccine, $\mathrm{VE}$ for vaccinees with an $\mathrm{ADCP}$ response above the median response magnitude was estimated to range from $62 \%$ to $79 \%$ and VE for vaccinees with an Env IgG3 breadth score above the median was estimated to range from $69 \%$ to $83 \%$, with identical estimates for Env $\mathrm{CD}^{+} \mathrm{T}$ cell PFS (Figure 4C). The causal inference method accounts for uncertainty in statistical inferences due to potential bias stemming from analyzing postrandomization subgroups (25). The 95\% estimated uncertainty interval surrounding these ranges of estimates for Env IgG3 breadth score, which accounts for uncertainty both due to sampling variability and to potential selection bias, is $43 \%$ to 97\% (Figure 4C). This result is remarkable in that it reveals the potential strength of antibody Fc effector function and Env IgG3 on impacting HIV-1 vaccine efficacy.

Human host genotype, Fc $\gamma$ RIII GA/AA, related to antibody-mediated phagocytosis correlated with reduced HIV-1 acquisition. Following screening (discussed in Methods), 7 single-nucleotide polymorphisms (SNPs) in the Fc $\gamma$ RIIa gene were chosen for assessing the effect of modification on the correlates of ADCP and Fc $\gamma$ RIIa binding with HIV-1 acquisition risk. Following corrections for multiple comparisons, an intron SNP (FCGR2A-intron13-645-G/A [rs2165088]) was found to modify the correlate of ADCP with HIV-1 acquisition risk (interaction $P$ value $=0.003$ and $Q$ value $=$ 0.006, Figure 5 and Table 6). Participants with the GG genotype had the same odds of HIV-1 acquisition regardless of ADCP (OR $=1.14, P=0.726$ ), while participants with GA/AA genotypes had an ADCP-associated reduced risk of HIV-1 acquisition $(\mathrm{OR}=0.16$, $P=0.0005)$. Notably, these findings withstood adjusting for cellular responses $(\mathrm{OR}=1.47, P=0.36$ for $\mathrm{GG}$ and $\mathrm{OR}=0.24, P=$ 0.005 for GA/AA, interaction $P$ value $=0.006$; Figure 5 and Table 6). That Fc $\gamma$ RIIa SNPs significantly modified the correlation of ADCP with HIV-1 infection risk demonstrates that the interplay of host FcR genetics in the population and vaccine-elicited immune responses can impact the outcome of HIV-1 vaccine efficacy trials. 
A

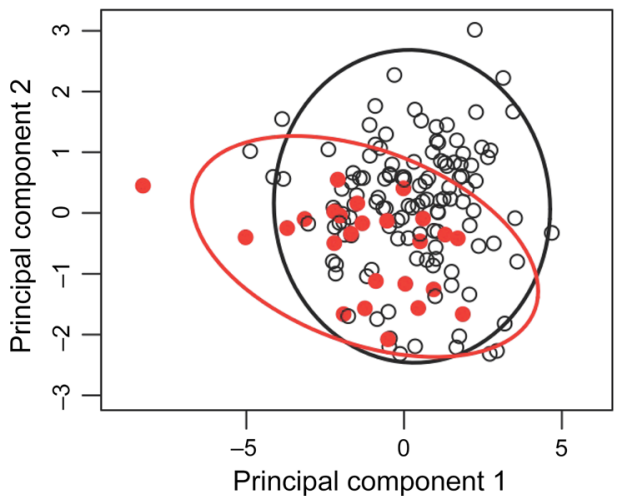

B

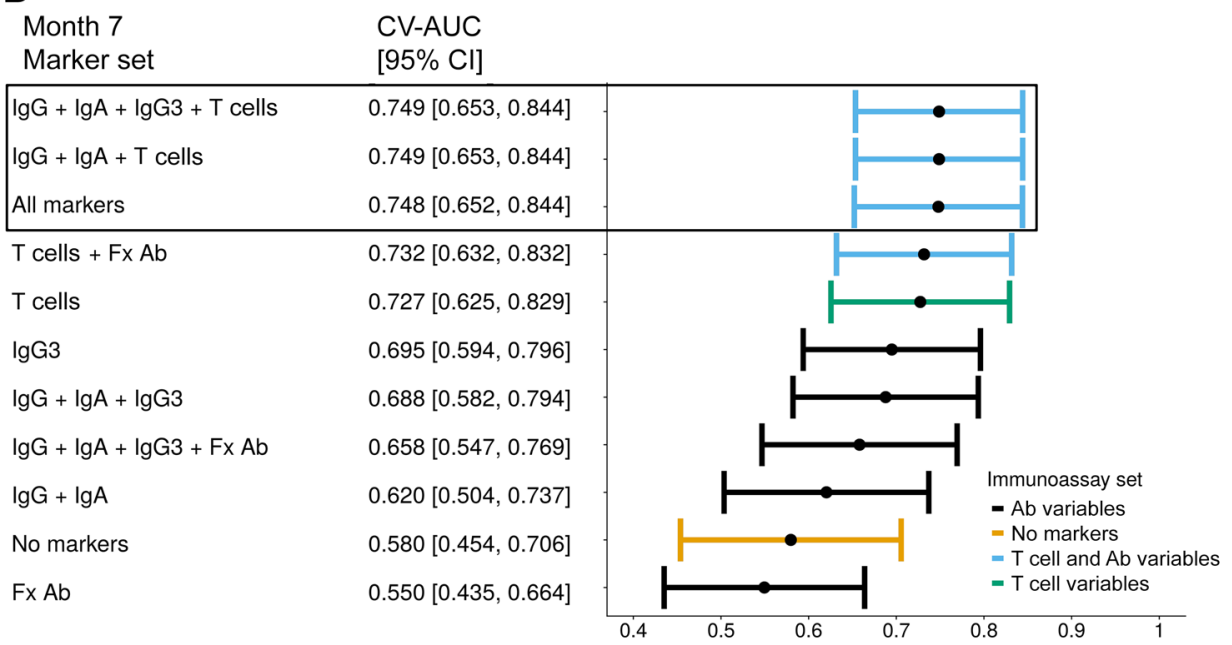

Major PC1 contributors ${ }^{\star \star}$

- High IgG Env

- High IgG gp41

- High ADCP

- High FcyR2a Env

- High IgG3 Env

Major PC2 contributors **

- High CD8 ${ }^{+}$polyfunctionality

- Low IgA Env

- High IgG3 Env
Although the SNP-effect modification on the correlate of FcyRIIa binding with HIV-1 acquisition risk was not significant (interaction $P$ value $=0.07$ ), the correlate of Fc $\gamma$ RIIa binding with HIV-1 acquisition risk was observed only in the vaccine recipients who carried GA/AA genotypes $(\mathrm{OR}=0.3, P=0.011$ or $\mathrm{OR}=0.48, P=$ 0.071 with adjusting for cellular responses). Similarly to ADCP and Fc $\gamma$ RIIa, the association of IgG3 and HIV-1 acquisition risk was significantly lower in the FCGR2A-intron13-645-GA/AA participants $(\mathrm{OR}=0.25, P=0.002)$. However, there was also a trend for the GG genotype ( $\mathrm{OR}=0.42, P=0.086$, unadjusted for cellular measurements) (Figure 5 and Table 6).

Antibody effector function significantly correlated with lower viral load setpoint in breakthrough infections. Although it is known that vaccine-elicited CD $8^{+} \mathrm{T}$ cells can exert selective pressure on HIV-1 infection in breakthrough human vaccinees (26), it is unknown if antibody Fc effector functions can play a protective role after acquisition in humans. We examined the relationship of the magnitude of antibody Fc effector functions with the viral load (VL) setpoint in those vaccinees who became infected (Table 7). VL setpoint was calculated as the mean plasma HIV-1 RNA level 10 to 20 weeks after diagnosis. Three regression models were performed for the 2 primary immune variables prespecified in the case control analysis in order to examine each one individually as well as in a combined model. While ADCP was found not to associate with VL setpoint in either model, Fc $\gamma$ RIIa correlated with lower VL setpoint in both the individual and combined models (Figure
6). Remarkably, there was an estimated $0.53 \log _{10}$ lower VL setpoint (95\% CI 0.06-1.00 $\log _{10}$ lower) per standard deviation (SD) increase in HIV-1 Env IgG binding to Fc $\gamma$ RIIa $(P=0.027)$. In separate models controlling for the same terms, IgG3 breadth and $\mathrm{CD} 8^{+} \mathrm{T}$ cell Env polyfunctionality were not observed to influence VL setpoint $(P=0.963$ and $P=0.565$, respectively).

\section{Discussion}

The correlates of protection for currently licensed vaccines in humans are predominantly attributed to IgG binding antibodies $(2,27,28)$. However, the underlying mechanisms of these pathogen-specific immune responses remain largely unknown. Critical to the advancement of improved vaccine regimens is determining mechanistic correlates of protection $(2,27,28)$. For pathogens like HIV-1 that lack an efficacious and licensed vaccine, identification of immune mechanisms of protection will inform practical improvements in vaccine immunogens toward eliciting protective responses. There is a sense of urgency for defining antibody mechanisms of protection to provide a roadmap for advancing the most promising vaccine candidates.

Vaccine-elicited antibody Fc effector functions were associated with decreased HIV-1 risk in human trials, including antibodydependent cell-mediated virus inhibition (ADCVI) in VAX004 (29) and ADCC in the presence of low plasma IgA in RV144 (18). We previously reported that no significant ADCC responses were elicited in HVTN 505 (6). However, given the FcrRIII association 


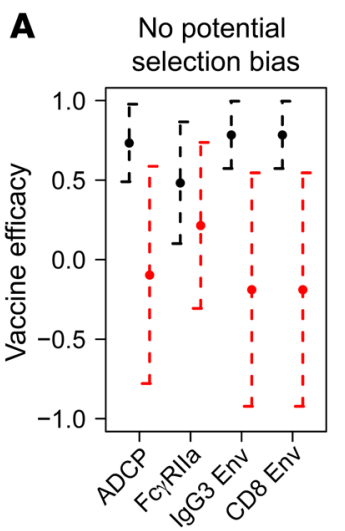

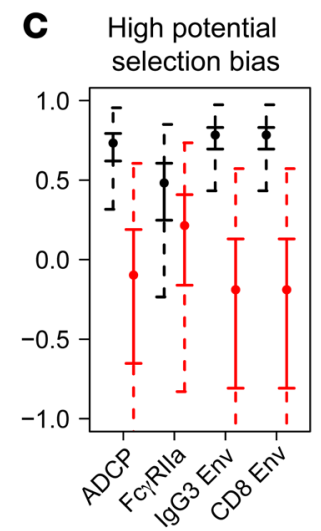

Figure 4. Antibody Fc effector function, IgG3, and $\mathrm{CD8}{ }^{+} \mathrm{T}$ cells stratify vaccinees into vaccine efficacy greater than zero and at (or near) zero. The ADCP immune measurement stratifies vaccinees into vaccine efficacy $>0$ (VE [1]) (black lines) and vaccine efficacy near 0 (VE [0]) (red lines), similar to CD8 $8^{+} \mathrm{T}$ cell Env polyfunctionality score. Ignorance intervals (solid lines) and 95\% estimated uncertainty intervals (EUIs) (dashed lines) for VE by subgroup of vaccinees were defined by cutting above and below the median response magnitude of each of the immune response measurements of ADCP, Fc $\gamma R$ Rlla, and CD8 Env method of Cilbert et al. (25). Each panel assumes no (A), medium (B), or high (C) potential selection bias. The amount of potential selection bias is specified by a model that varies defined sensitivity parameters over the range $[0,0],[-0.5,0.5]$, or $[-1,1]$, respectively. $n=25$ infected and 125 uninfected participants. with decreased risk of HIV-1 acquisition in this study, further studies with ADCC methods that include HIV-1 isolates from infected vaccinees and placebos could provide improved detection of these responses. Moreover, in nonhuman primate models of HIV, Env-specific IgG ADCP correlated or associated with delayed acquisition from virus challenge (8-12). Additionally, in nonhuman primate studies, non-neutralizing antibodies were associated with decreased VL after infection (30-32) and limiting founder viruses (33). Phagocytic activity is mediated by antibody immune complex signaling through Fc $\gamma$ RIIa on effector cells $(1,34)$. Consequently, ADCP and FcR engagement are emerging hypotheses that can be tested as a protective antibody mechanism upon completion of the ongoing vaccine efficacy trials including HVTN 702 and 705 (11, 35). Fc $\gamma \mathrm{R}$ interactions with Fc regions of antibodies leading to antiviral functions depend on a variety of factors including antigen specificity, antibody isotype and subclass (19), Fc region glycosylation $(1,36)$, allosteric interactions upon immune complex formation $(37,38)$, and FcR genetic polymorphisms (39). An efficient method to capture both precise antigen specificity and functional activity through $\mathrm{Fc} \gamma \mathrm{R}$ is via a combined approach of a multiplexed Fc $\gamma \mathrm{R}$ binding assay to rapidly quantify $\mathrm{Fc} \gamma \mathrm{R}$ engagement of individual antibody specificities in polyclonal specimens (40) and measuring phagocytosis of antigen-specific antibody immune complexes by monocytes (i.e., ADCP) $(19,41)$.

In this most recently completed HIV-1 vaccine efficacy study, we sought to examine the role of ADCP and Fc $\gamma$ RIIa engagement to evaluate antibody effector functions underlying IgG binding correlates of risk. We prespecified a primary hypothesis on the blinded case control analysis that HIV-1 Env glycoprotein-specific Fc $\gamma$ RIIa engagement and ADCP function would inversely correlate with HIV-1 acquisition risk. Our primary hypothesis was supported in that Env-specific Fc $\gamma$ RIIa activity and ADCP each correlated significantly with decreased HIV-1 risk in HVTN 505 (OR = 0.48, $P<0.001$ and $\mathrm{OR}=0.47, P=0.001$, respectively) and remained significant after correction for $\mathrm{CD}^{+} \mathrm{T}$ cell polyfunctionality. To the best of our knowledge, these are the first and only antibody-mediated functional correlates of risk for HVTN 505 to date.

Unexpectedly, we also observed that the risk of HIV-1 infection was dramatically lower in those vaccinees with low/undetectable serum HIV-1 Env IgA $(\mathrm{OR}=0.16$ and $0.37, P=0.006$ and $P<0.001)$ for ADCP and Fc $\gamma$ RIIa, respectively. Although there was no direct impact of serum Env IgA on infection risk in HVTN
A

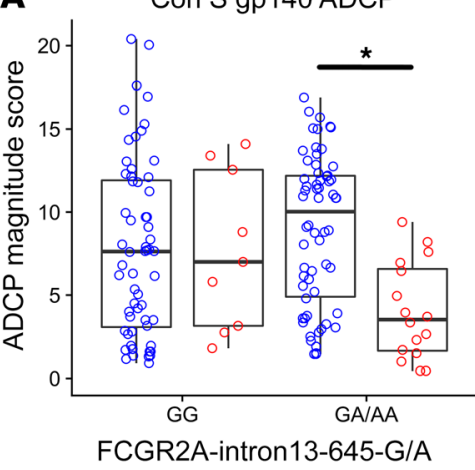

B

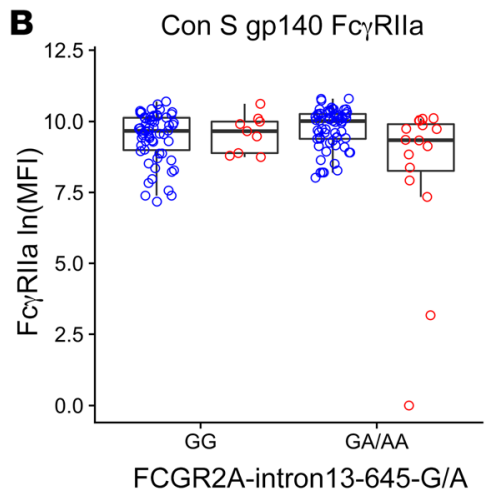

C

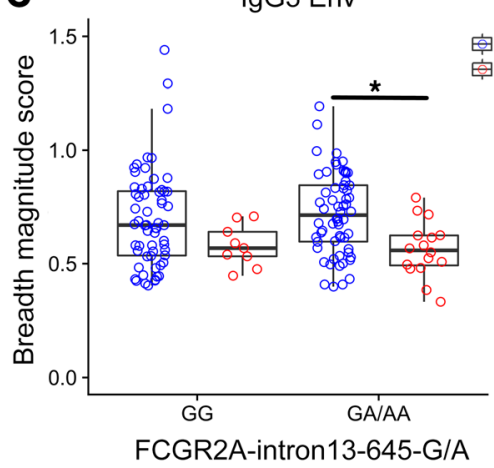

Figure 5. Host genetics (Fc $\gamma$ RIla SNPs) significantly modified the correlation of ADCP with HIV-1 infection risk. ${ }^{* *}$ Interaction $P=0.0006$ and $Q$ value $=$ 0.026 , *within genotype group $P<0.05$ after adjusting for all covariates and 2 cellular variables. $P$ values were calculated based on the Wald test. Con $S$

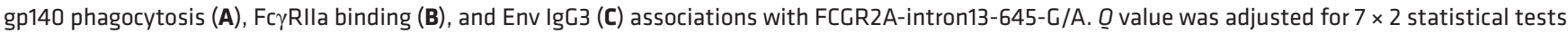
with respect to 7 FcyRlla SNPs and 2 primary Fc effector functions. In the first analysis, we adjusted for age, race, behavior risk, and BMI. In the second analysis, in addition to adjusting these covariates, we also adjusted for 2 cellular responses, Env CD4+ $T$ cell polyfunctionality score (PFS) and Env CD8 ${ }^{+} T$ cell PFS. Box-and-whisker plots show the median line and interquartile ranges. There are 9 infected and 60 uninfected vaccinees with the GG genotype and 16 infected and 62 uninfected in the GA/AA genotype group. 
Table 6. Host genetic analysis of risk for HIV-1 acquisition among HVTN 505 participants

\begin{tabular}{|c|c|c|c|c|c|}
\hline & & & $\mathrm{OR}$ & $P$ & Int. $P^{A}$ \\
\hline \multirow{4}{*}{$\mathrm{ADCP}$} & & GG & 1.14 & 0.726 & 0.003 \\
\hline & & GA/AA & 0.16 & 0.0005 & \\
\hline & Adj. cell ${ }^{\mathrm{B}}$ & GG & 1.47 & 0.361 & 0.006 \\
\hline & & GA/AA & 0.24 & 0.005 & \\
\hline \multirow[t]{4}{*}{ FcyRlla } & & GG & 1.11 & 0.849 & 0.072 \\
\hline & & GA/AA & 0.3 & 0.011 & \\
\hline & Adj. cell ${ }^{\mathrm{B}}$ & GG & 1.5 & 0.517 & 0.112 \\
\hline & & GA/AA & 0.48 & 0.071 & \\
\hline \multirow[t]{4}{*}{$\operatorname{lgG3}$} & & GG & 0.42 & 0.086 & 0.444 \\
\hline & & GA/AA & 0.25 & 0.002 & \\
\hline & Adj. cell ${ }^{B}$ & GG & 0.51 & 0.21 & 0.368 \\
\hline & & GA/AA & 0.26 & 0.007 & \\
\hline
\end{tabular}

AInteraction assesses for an interaction between Fc $\gamma$ RIla genotypes and the variable tested. ${ }^{B}$ Cellular adjustment adjusts for previously published CD4 and CD8 polyfunctionality scores.

505 (6), this finding is consistent with the hypothesis that emerged from the RV144 HIV-1 vaccine trial that Env IgA circulating in the blood was either a surrogate for another response associated with increased HIV-1 risk or that Env IgA could compete with Env IgG for Fc effector functions $(17,18)$. It is important to note that antiviral functions of circulating IgA are likely different from antiviral functions of mucosal $\operatorname{IgA}$, and that other antiviral IgA mechanisms $(19,42-45)$ not tested here could be involved in protective efficacy. Given our systems immunology analysis, a possible synergistic effect between Fc $\gamma$ RIIa binding, ADCP, IgG3, serum IgA, and $\mathrm{CD}^{+} \mathrm{T}$ cells may also explain potential protection; absence of serum IgA may enable FcyRIIa-mediated phagocytosis to eliminate infectious virions and/or cells in parallel with $\mathrm{CD}^{+} \mathrm{T}$ cellmediated antiviral functions such as virus inhibition elicited by this vaccine regimen (46) and/or through mechanisms associated with the polyfunctional responses by these vaccine-elicited CD8 ${ }^{+}$ T cells (5). The observed heterogeneity in vaccine-elicited immune responses among vaccine recipients and the distinct immune measurements that correlate with HIV-1 acquisition suggest that low levels of one protective immune response may be compensated for by the presence of another protective immune response.
Notably, of the humoral responses, ADCP and IgG3 were among the strongest correlates of decreased HIV-1 acquisition; however, Fc $\gamma$ RIIa engagement was the only correlate of decreased VL in infected vaccinees. We previously reported that vaccine-elicited Env IgG3 had a short response half-life (47), so the lack of correlation with VL could be due to the vaccine-elicited IgG3 decline by the time of infection. Alternatively, different sets of humoral responses could be responsible for prevention of infection versus control of viremia once infected. This suggests that HIV vaccines that induce a combination of humoral responses including ADCP, Env IgG3, and antibodies that engage Fc $\gamma$ RIIa can impact both or either virus acquisition and VL setpoint.

Given that Fc $\gamma$ RIIa binding correlated with HIV-1 risk in this study and that host FcR genetic polymorphisms can directly influence antibody Fc effector functions at the individual level in vivo, we then asked whether polymorphisms in Fc $\gamma$ RIIa genes impacted the correlation of antibody functions with HIV-1 risk. We found that Fc $\gamma$ RIIa genotype in association with vaccine-elicited antibody responses significantly correlated with HIV-1 acquisition risk, providing a profound link between circulating vaccine-elicited antibodies and the impact of individual genetic variation in effector cells that can serve to engage vaccine-elicited responses for protective efficacy. There is a precedent for other FcR polymorphisms playing a role in HIV-1 acquisition risk in vaccine efficacy studies (48). In this study, ADCP- and Fc $\gamma$ RIIa binding-associated reduction in risk of HIV-1 acquisition was only evident in individuals with the GA/AA SNP, while an IgG3 Env-associated reduction in risk also trended with the GG SNP when unadjusted for $\mathrm{CD}^{+}$and $\mathrm{CD} 8^{+}$ T cell polyfunctionality. Like all intron SNP associations, these findings may be explained through random chance, altered expression levels of the associated gene, splice variants in the associate protein, or in linkage disequilibrium with a causal variant outside of the considered regions. Certain Fc $\gamma \mathrm{R}$ haplotypes have also been associated with enhanced rate of infection (49). Therefore, future studies should test replication of these findings in another cohort by directly examining these FCR SNPs and interrogating autologous FcRexpressing effector cells with vaccine-elicited antibodies in immune correlates analyses of vaccine efficacy trials. To further interrogate potency and breadth of mechanisms of protection, recent HIV-1 sequences circulating in the population need to be included in assays as part of the evaluation of correlates of protection $(50,51)$. Although we examined vaccine-matched Env among other diverse

Table 7. Linear regression models looking at the association between $\log _{10}$ viral load setpoint and the primary immune measurements

\begin{tabular}{|c|c|c|c|c|c|c|c|c|c|}
\hline \multirow[b]{2}{*}{ Analysis } & \multicolumn{3}{|c|}{ Model 1} & \multicolumn{3}{|c|}{ Model 2} & \multicolumn{3}{|c|}{ Model 3} \\
\hline & Est. & Std error & $95 \% \mathrm{Cl}$ & Est. & Std error & $95 \% \mathrm{Cl}$ & Est. & Std error & $95 \% \mathrm{Cl}$ \\
\hline Hispanic/Other (non-White) & -1.25 & 0.106 & $-2.77,0.27$ & -1.12 & 0.103 & $-2.46,0.22$ & -1.14 & 0.109 & $-2.55,0.26$ \\
\hline White & -0.08 & 0.901 & $-1.35,1.19$ & -0.32 & 0.596 & $-1.49,0.86$ & -0.36 & 0.573 & $-1.61,0.89$ \\
\hline $\mathrm{ADCP}$ & -0.27 & 0.253 & $-0.74,0.20$ & & & & -0.06 & 0.797 & $-0.52,0.40$ \\
\hline Fc $\gamma$ Rlla & & & & -0.55 & $0.011^{\AA}$ & $-0.98,-0.12$ & -0.53 & $0.027^{\mathrm{A}}$ & $-1.00,-0.06$ \\
\hline
\end{tabular}

Race (non-White [Hispanic/other] and White) and the indicator of whether diagnosis was made prior to seroconversion are adjusted (pre-seroconversion). The Ab functional markers are scaled to have a standard deviation of 1. Estimates, standard errors, and $95 \%$ confidence intervals are shown. ${ }^{A}$ Significant impact of marker on VL setpoint is indicated in bold. Model 3 is adjusted for both biomarkers. 
A
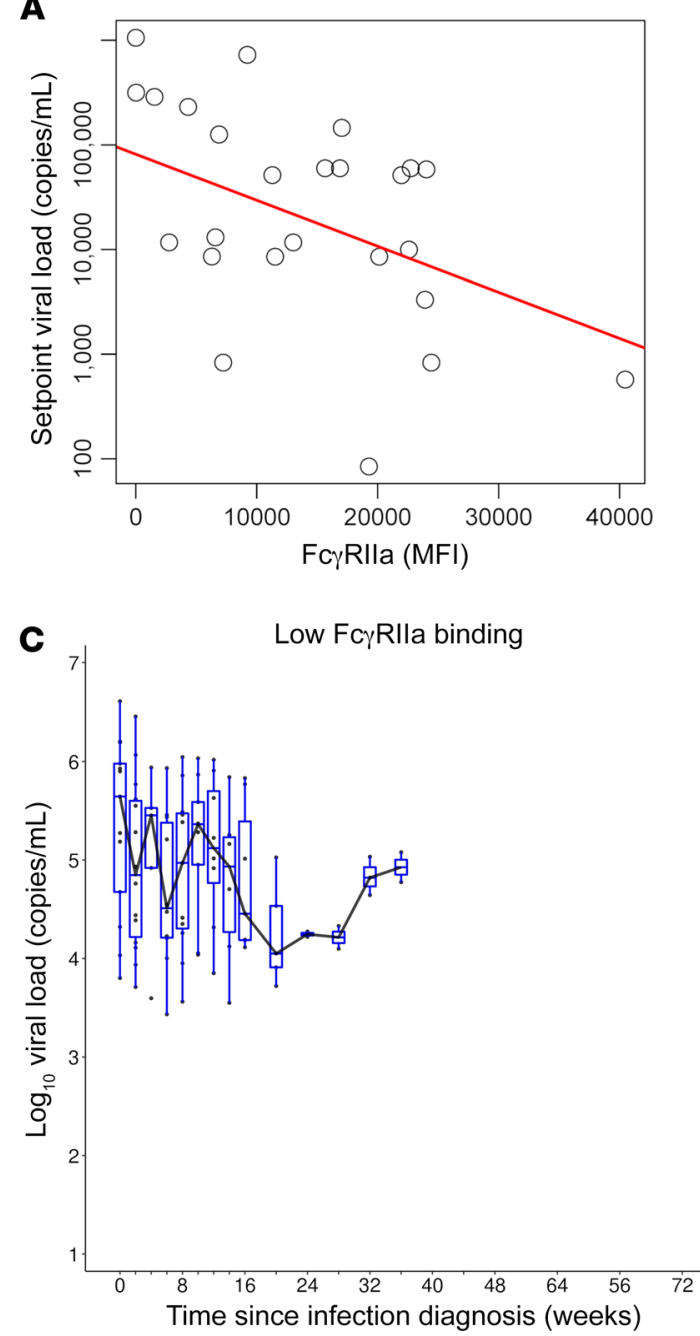

B

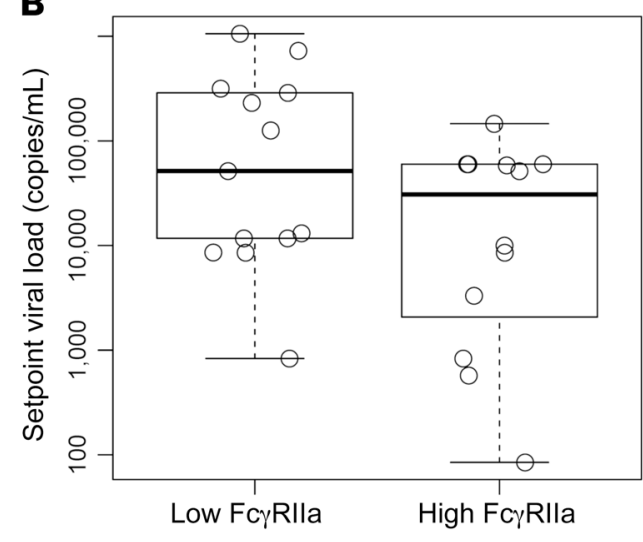

High Fc $\gamma$ RIla binding

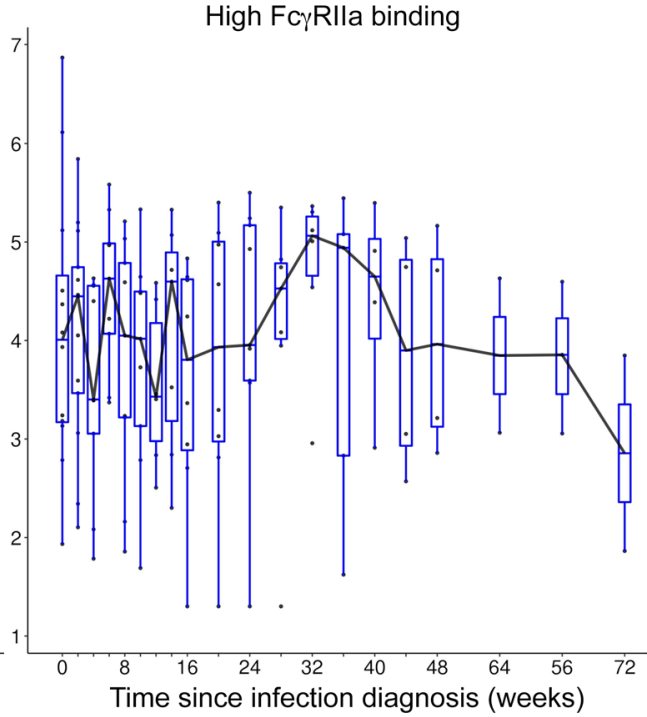

Figure 6. Host setpoint viral load negatively correlates with Fc $\gamma$ RIla binding. Fc $\gamma$ RIla and representative imputed viral load setpoint (A and $\mathbf{B}$ ) and observed viral load (measured as RNA copies/mL) (C) are displayed. Each standard deviation increase in Fc $\gamma$ RIla associated with a $0.53 \log _{10}$ viral load decrease in viral load setpoint $(P=0.027,95 \% \mathrm{Cl}=0.06$ to 1.00 , linear regression shown in $\mathbf{A}$ ). Dichotomized cases and viral load are displayed (B), with time since infection and median in C. For FcyRlla low-binding participants, $n=13$ (at baseline), 14 (at week 2), 5 (at week 4), 10 (at week 6), 11 (at week 8), 9 (at week 10), 8 (at week 12), 6 (at week 14), 7 (at week 16), 5 (at week 20), 2 (at week 24), 2 (at week 28), 2 (at week 32), and 2 (at week 36). For Fc $\gamma$ Rlla high-binding participants, $n=12$ (at baseline), 13 (at week 2), 9 (at week 4), 9 (at week 6), 8 (at week 8), 9 (at week 10), 6 (at week 12), 7 (at week 14), 8 (at week 16), 8 (at week 20), 9 (at week 24), 7 (at week 28), 6 (at week 32), 5 (at week 36), 4 (at week 40), 4 (at week 44), 4 (at week 48), 2 (at week 64), 3 (at week 56), and 2 (at week 72). Box-and-whisker plots show the median line and interquartile ranges. $P$ values were calculated using the Wald test. proteins for $\mathrm{F} c \gamma \mathrm{R}$ analysis, we selected the consensus HIV-1 Env glycoprotein, Con S gp140, as part of the primary hypothesis for both FcR and ADCP analysis, since inherently as a consensus sequence it may best represent diverse strains of Env. This selection was also informed by our prior analysis demonstrating an independent role for Con S gp140 IgG in modulating HIV-1 acquisition risk in HVTN 505 , an effect which was not observed in any clade B gp140 examined, including both vaccine (VRC B gp140) and consensus (B.con. env03 gp140) (6). Antibodies that bind HIV-1 Env on intact infectious virions as well as on the surface of infected cells are strong candidates for effective antiviral Fc effector function, through their recruitment of cellular FcRs. Further immune correlates analyses to test antibody $\mathrm{Fc}$ effector functions would be strengthened by examining HIV-1 virus sequences from infected vaccinees $(4,52)$ to examine whether Fc $\gamma$ RIIa binding and ADCP directly exerted immune pressure and selectively eliminated certain sequences from being transmitted. Immune pressure by non-neutralizing antibodies of the type elicited by the HVTN 505 trial (i.e., anti-gp41 $\mathrm{IgG}$ ) was reported in a humanized mouse model (53), indicating the potential of vaccine-elicited non-neutralizing antibodies to control infection in humans.

One limitation of our study, which can be examined in future analyses, is to examine HVTN 505 breakthrough virus sequences as part of the reagents in the humoral assays. The immune mechanism(s) driving the HVTN 505 sieve effect (4) is unknown; however, the findings from this current study suggest Env IgG3 breadth and Fc effector functions (i.e., ADCP, Fc $\gamma$ RIIa engagement) of antibodies directed against specific Env sieve sites as a possibility.

A comprehensive analysis of humoral and cellular responses revealed the different roles of humoral and cellular immunity in HIV-1 risk and revealed that among the functional immune responses examined, ADCP, Env IgG3, and CD8 ${ }^{+} \mathrm{T}$ cell polyfunctionality are strong modifiers of vaccine efficacy. This study also revealed the role of $\mathrm{FCR}$ host genetics in modifying vaccine efficacy by antibody Fc effector functions. An additional important finding from this study is the evidence of postinfection control of viremia, lower VL setpoint, associated with the magnitude of IgG engagement of Fc $\gamma$ RIIa. Since the study was not designed to sample acute infection, insufficient samples were collected during the acute phase to reliably observe peak VL across participants. Antibody Fc effector functions were associated with VL control in vaccinated rhesus macaques (30-32) and the data presented in this report provide evidence in humans that functional antibodies, specifically Env gp140 antibodies that engage Fc $\gamma$ RIIa elicited by vaccination, may impact viremia. Conceptually, antibody-mediated Fc effector functions could work in a number of ways to prevent 
systemic HIV-1 infection, including clearance of virions that may decrease the number of functionally infectious virions to infect new cells and phagocytosis or trogocytosis of infected cells (54, 55) that may limit their capacity to produce and spread new virions. Although our analysis showed no impact from IgG3, timing of exposure may be important to any impact on VL due to the rapid decline of Env IgG3 following vaccination (47).

The findings from this study underscore the importance of correlates-based immunogen design and suggest that a potential target for HIV vaccine strategies is the elicitation of IgG ADCP and Fc $\gamma$ RIIa. Insights into effective strategies for inducing specific HIV epitope-reactive, Fc $\gamma$ R-reactive, and functional antibodies are emerging from preclinical and clinical trial comparisons of different adjuvants and prime-boost strategies. Further work to understand the biological role of antibody Fc effector functions in vivo and how to best measure these functions in clinical trials is critical for informing immunogen design aimed to improve the breadth, magnitude, and population coverage of future HIV-1 vaccines. Designing and testing immunogen regimens that reliably induce immune correlates of interest will allow future vaccine efforts to focus on honing immune responses to optimal protection and to determine if they are correlates of protection. The results reported here on antibody effector functions and HIV-1 risk provide strong rationale to test ADCP, Env IgG3, and FcR engagement as mechanistic immune correlates of protection in upcoming vaccine efficacy trials.

\section{Methods}

HVTN 505 trial. The HVTN 505 clinical trial was previously described and participants (Clinicaltrials.gov ID: NCT00865566) provided written informed consent $(3,6)$. Immune responses to vaccination were assessed at 7 months, which was 4 weeks after the final vaccination, in 25 primary endpoint vaccine cases (diagnosed with HIV-1 infections between month 7 and month 24), and 125 randomly sampled frequencymatched vaccine controls (HIV-1 negative at month 24).

$A D C P$. ADCP was performed as previously described $(19,41)$. Briefly, quantification of ADCP was performed by covalently binding Con S gp140 Env glycoprotein to fluorescent beads and forming immune complexes by incubating in the presence of 1:50 dilution of serum. Immune complexes were then incubated in the presence of THP-1 cells (human-derived monocyte line, ATCC TIB-201), and the fluorescence of the cells was detected using flow cytometry (BD LSRII). The magnitude of the ADCP immune response was calculated as an ADCP score by multiplying the mean fluorescence intensity (MFI) and frequency of phagocytosis-positive cells and dividing by the MFI and frequency of the bead-positive cells in an antibody-negative (PBS) control well. To assess sensitivity, 2 independent experiments were conducted and analyzed independently with similar results for correlates of HIV-1 risk. CH31 mAb (Catalent, catalog 3412, $25 \mu \mathrm{g}$ / $\mathrm{mL}$ ), a CD4bs broadly neutralizing antibody (56), was included as a positive control and the non-HIV-specific CH65 IgG1 mAb (Catalent, catalog 3369, $25 \mu \mathrm{g} / \mathrm{mL}$ ) (57) was included as a negative control.

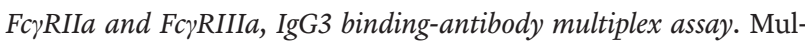
tiplexed human Fc receptor binding assays were a modified version of the binding-antibody multiplex assay (BAMA) $(17,20,58,59)$ and adapted from previously described methods $(36,40,60)$. Avi-tagged high-affinity FcR proteins, Fc $\gamma$ RIIa H131 and Fc $\gamma$ RIIIa F158, were provided by Hua-Xin Liao, James Peacock, and Barton Haynes (Duke
Protein Production Facility). Briefly, antigen-coupled beads were incubated in the presence of 1:50 vaccinee serum, followed by a detection tetramer constructed from biotinylated Fc $\gamma$ Rs and streptavidin-PE. Fluorescence intensity was resolved using the flow-based Luminex 3D Platform. Positive controls included polyclonal HIVIG (provided by the NIH AIDS Reagent Program), VRC01 IgG1 mAb (provided by John Mascola, Vaccine Research Center, NIAID), and CH58 IgG1 mAb (provided by Hu-Xin Liao and Barton Haynes, Duke University [ref. 61]). HIV-1-negative human serum (MilliporeSigma) served as a negative control, and bead binding was controlled by subtracting the fluorescence of blank or MulVgp70-coated beads. HIV-1 Env glycoproteins tested include the vaccine strains (VRC A gp140, VRC B gp140, and VRC C gp140), and a cross-clade panel of consensus gp140 proteins (A1con.env03 CF, B.con.env03 140 CF, C.con.env03 140 CF); consensus gp120 and gp140 proteins (Con 6 gp120, Con S gp140CFI); V1V2 proteins (AE.A244 V1V2 Tags, C.1086 V2 Tags, C.1086 V1V2 Tags, VRC A gp70V1V2 [provided by Gary Nabel, Vaccine Research Center, NIAID], gp70_B.CaseA2 V1/V2, gp70_B.CaseA2 V1/V2/169K, and gp70_C.1086 V1/V2) (20, 61); gp41 (clade B, Immunodiagnostics); p24 Gag (clade B, BD Biosciences); and B.MN V3 gp70. Each experimental condition was performed in duplicate, and the results were repeated and confirmed by a second operator in an independent experiment.

Fc $\gamma$ R SNP genotyping. A total of 193 samples, DNA from the peripheral blood mononuclear cells, from 145 uninfected vaccinees (defined as HIV negative at month 24) and 48 infected vaccinees (defined as becoming HIV infected sometime between week 28 and month 24) were genotyped for FCGR2A as previously described (48). Indels, double mutations, and SNPs that did not pass the minor allele frequency threshold $(\geq 5 \%)$ and the Hardy-Weinberg equilibrium test $(P$ $\geq 0.00001$ ) based on the control samples were excluded. After exclusion, 7 SNPs remained for this analysis.

Statistics. To evaluate HIV-1 Env ADCP and log-transformed Fc $\gamma$ RBAMA fluorescence intensities measured at month 7 among vaccinees as correlates of HIV-1 infection through month 24, based on follow-up data collected prior to study unblinding on April 22, 2013, logistic regression methods (62) accounting for the nested case-control biomarker sampling design were used (osDesign R package, Comprehensive Archive R Network). Pseudo likelihood-based inference methods were used to evaluate statistical significance. Before regression analysis, immune response variables were mean-centered and SD-scaled (based on distributions among vaccinees); thus, a unit change in the variable represents a change of $1 \mathrm{SD}$. All regression models included the following baseline covariates to adjust for potential confounding: participant age, race (White vs. Black vs. Hispanic/Other), BMI, and a behavioral risk score $(3,6)$. Regression hypotheses were structured a priori into a hierarchy of primary, secondary, and exploratory tiers based on scientific importance. To account for multiple testing, $P$ values were adjusted to form $Q$ values by controlling for false discovery rate (63). For a sensitivity analysis of the influence of the 2 vaccinees with Fc $\gamma$ RIIa binding below $4 \ln (\mathrm{MFI})$, we truncated their values to 6 , a value much closer to the bulk of the data (Figure 1). A P value less than 0.05 was considered statistically significant. The association of Fc $\gamma$ RIIa binding with HIV-1 risk remained significant ( $\mathrm{OR}=0.54, P=0.002)$; however, adjustment for $\mathrm{CD} 8^{+} \mathrm{T}$ cell polyfunctionality in the sensitivity analysis increased the $\mathrm{OR}$ and $P$ value $(\mathrm{OR}=0.72, P=0.113)$. The association of Fc $\gamma$ RIIa binding with HIV-1 risk also remained significant in the IgA interaction model $\left(\mathrm{OR}=0.405\left[\operatorname{IgA} A^{-}\right], P=0.001 ; \mathrm{OR}=0.682\left[\operatorname{IgA}^{+}\right], P=0.002\right)$ with the interaction $P$ value being 0.200 . Env IgG3 breadth is a weight- 
ed combination of the Env immune response measurements (consensus gp140 Env glycoproteins for subtypes A1, B, and C, the vaccine-matched Env glycoproteins [VRC A, VRC B, and VRC C], group M Con S gp140, and Con 6 gp120), where the weights are the product of variance standardization weights and tree-based diversity maximization weights (18). This is the same procedure used for the score variables in Fong et al. (6).

Ignorance intervals were calculated as previously described (64). All analyses were performed in the R programming system (https:// cran.r-project.org/). SuperLearner, an ensemble, loss-based, cross-validated statistical method for developing predictive models (24), included all immune measurements with a positive response call, greater than $20 \%$ vaccine group response rate, and a greater response in vaccine versus placebo group. Measurements that were highly correlated (Spearman's rho > 0.9) and that had a low dynamic range across vaccinees were not included. All models included the baseline variables of age, BMI, and baseline risk score. The measurements included in the SuperLearner analysis were specified in a plan before analysis (see supplemental material).

$V L$ analysis. The association of antibody function with VL setpoint was performed on the 25 cases (diagnosed with HIV-1 infection) in the vaccine arm. Table 7 includes results from 3 linear regression models, one for each of the 2 primary variables and one adjusted for both biomarkers. The standard errors take into consideration uncertainties resulting from imputation with the help of R package mitools. For the 5 vaccinees with missing VL data, 100 imputed data sets were developed. The variables to impute missing VL setpoint values included age, race, baseline behavioral risk score, diagnosis year, number of DNA vaccinations, indicator of Ad5 vaccination, Env-specific CD8 ${ }^{+} \mathrm{T}$ cell PFS (5), BMI, an indicator of whether diagnosis was made prior to seroconversion, $\log \mathrm{VL}$ at diagnosis, and early VL.

Study approval. All participants in HVTN 505 (ClinicalTrials.gov ID: NCT00865566) provided written and informed consent prior to inclusion in the study as reported (3). Testing of HVTN 505 samples reported here were approved through the Institutional Review Board at Duke University (IRB Pro00004500, Immunologic and Biologic Testing of HVTN Specimens).

\section{Author contributions}

SDN, YF, JM, GF, PBG, and GDT designed the research study. SDN, YF, SSL, DEG, BDW, WCY, KES, XS, SS, LZ, ACD, BSB, MS, CWP,
HEJ, and RG processed and analyzed the data. KES, NLY, and XS supervised the work, SDN, DEG, DG, FF, and CWP conducted experiments. IF, STK, EMS, JRM, BSG, SMH, MES, and LC designed and performed the clinical trials. SDN, YF, PBG, and GDT wrote the manuscript and all coauthors edited and reviewed the manuscript. The first author position is shared by SDN, YF, and SSL for their substantial contributions: SDN led humoral experimental design and drafted the manuscript, YF led analysis efforts for humoral experiments, and SSL led analysis for host genetic findings. The order of these first authors is based on when work on this study began.

\section{Acknowledgments}

We are indebted to the HVTN 505 volunteers and staff for participation and successful completion of the HIV-1 efficacy trial. We thank the HVTN 505 Clinical Team: Susan Buchbinder, Edwin DeJesus, Ian Frank, Spyros Kalams, Mike Keefer, Mark Mulligan, and Richard Novak. See Supplemental Acknowledgments for HVTN 505 Team details. We thank Judith Lucas, Michael Archibald, Kasey Hernandez, and Lawrence Armand for expert technical assistance; Marcella Sarzotti-Kelsoe and the Quality Assurance Unit (QAU) for quality assurance oversight; Kristy Long, David Beaumont, Sahill Patel, and April Randhawa for expert data and program management; Jamie Peacock and the Duke Human Vaccine Institute Protein Production Facility for proteins; and Steven Walsh for helpful discussion. This work was supported by NIAID US Public Health Service grants UM1 AIO68614 (LOC: HIV Vaccine Trials Network), UM1 AI068618 (LC: HIV Vaccine Trials Network), UM1 AI068635 (SDMC: HIV Vaccine Trials Network), NIH/NIAID P01 AI120756, and the NIH/NIAID Duke Center for AIDS Research (AI064518). MJM, GF, PBG, and GDT are recipients of funding from the Bill and Melinda Gates Foundation. GDT is an NIH AIDS Vaccine Advisory Committee Member and serves on advisory boards for NIH NIAID-funded grants and consortia. The content is solely the responsibility of the authors and does not necessarily represent the official views of the NIH.

Address correspondence to: Georgia D. Tomaras, Room 4079 MSRBII, 2 Genome Ct., Duke University, Durham, North Carolina 27710, USA. Phone: 919.681.5598,Email: gdt@duke.edu.
1. Bournazos S, Ravetch JV. Fc $\gamma$ receptor function and the design of vaccination strategies. Immunity. 2017;47(2):224-233.

2. Tomaras GD, Plotkin SA. Complex immune correlates of protection in HIV-1 vaccine efficacy trials. Immunol Rev. 2017;275(1):245-261.

3. Hammer SM, et al. Efficacy trial of a DNA/ rAd5 HIV-1 preventive vaccine. $N$ Engl J Med. 2013;369(22):2083-2092.

4. deCamp AC, et al. Sieve analysis of breakthrough HIV-1 sequences in HVTN 505 identifies vaccine pressure targeting the CD4 binding site of Envgp120. PLoS ONE. 2017;12(11):e0185959.

5. Janes HE, et al. Higher T-cell responses induced by DNA/rAd5 HIV-1 preventive vaccine are associated with lower HIV-1 infection risk in an efficacy trial. J Infect Dis. 2017;215(9):1376-1385.

6. Fong $\mathrm{Y}$, et al. Modification of the association between T-cell immune responses and human immunodeficiency virus type 1 infection risk by vaccine-induced antibody responses in the HVTN 505 Trial. J Infect Dis. 2018;217(8):1280-1288.

7. Nimmerjahn F, Ravetch JV. Antibody-mediated modulation of immune responses. Immunol Rev. 2010;236:265-275.

8. Bradley T, et al. Pentavalent HIV-1 vaccine protects against simian-human immunodeficiency virus challenge. Nat Commun. 2017;8:15711.

9. Barouch DH, et al. Protective efficacy of adenovirus/protein vaccines against SIV challenges in rhesus monkeys. Science. 2015;349(6245):320-324.

10. Barouch DH, et al. Protective efficacy of a global HIV-1 mosaic vaccine against heterologous SHIV challenges in rhesus monkeys. Cell. 2013;155(3):531-539.

11. Barouch DH, et al. Evaluation of a mosaic HIV-1 vaccine in a multicentre, randomised, doubleblind, placebo-controlled, phase $1 / 2$ a clinical trial (APPROACH) and in rhesus monkeys (NHP 13-19). Lancet. 2018;392(10143):232-243.

12. Ackerman ME, et al. Route of immunization defines multiple mechanisms of vaccine-mediated protection against SIV. Nat Med. 2018;24(10):1590-1598.

13. Watzl C, Long EO. Signal transduction during activation and inhibition of natural killer cells. Curr Protoc Immunol. 2010; Chapter 11:Unit 11.9B.

14. Zhang Y, Hoppe AD, Swanson JA. Coordination of Fc receptor signaling regulates cellular commitment to phagocytosis. Proc Natl Acad Sci U S A. 2010;107(45):19332-19337.

15. Dugast AS, et al. Decreased Fc receptor expression on innate immune cells is associated with impaired antibody-mediated cellular phagocytic activity in chronically HIV-1 infected individuals. Virology. 2011;415(2):160-167.

16. Bhatnagar N, et al. Fc $\gamma$ RIII (CD16)-mediated ADCC by NK cells is regulated by mono- 
cytes and Fc $\gamma$ RII (CD32). Eur JImmunol. 2014;44(11):3368-3379.

17. Haynes BF, et al. Immune-correlates analysis of an HIV-1 vaccine efficacy trial. N Engl J Med. 2012;366(14):1275-1286.

18. Tomaras GD, et al. Vaccine-induced plasma IgA specific for the $\mathrm{C} 1$ region of the HIV-1 envelope blocks binding and effector function of IgG. Proc Natl Acad Sci U S A. 2013;110(22):9019-9024.

19. Tay MZ, et al. Antibody-mediated internalization of infectious HIV-1 virions differs among antibody isotypes and subclasses. PLoS Pathog. 2016;12(8):e1005817.

20. Zolla-Pazner S, et al. Vaccine-induced IgG antibodies to V1V2 regions of multiple HIV-1 subtypes correlate with decreased risk of HIV-1 infection. PLOS ONE. 2014;9(2):e87572.

21. Neidich SD, et al. Increased risk of influenza among vaccinated adults who are obese. Int $J$ Obes (Lond). 2017;41(9):1324-1330.

22. Averhoff F, Mahoney F, Coleman P, Schatz G, Hurwitz E, Margolis H. Immunogenicity of hepatitis B Vaccines. Implications for persons at occupational risk of hepatitis $B$ virus infection. Am J Prev Med. 1998;15(1):1-8.

23. Ingardia CJ, Kelley L, Steinfeld JD, Wax JR. Hepatitis $B$ vaccination in pregnancy: factors influencing efficacy. Obstet Gynecol.1999;93(6):983-986.

24. van der Laan MJ, Polley EC, Hubbard AE. Super learner. Stat Appl Genet Mol Biol. 2007;6:Article25.

25. Gilbert P, Blette B, Shepherd B, Hudgens M. Post-randomization biomarker effect modification in an HIV vaccine clinical trial [preprint]. Posted on ArXiv November 9, 2018. https://arxiv. org/abs/1811.03930.

26. Rolland $\mathrm{M}$, et al. Genetic impact of vaccination on breakthrough HIV-1 sequences from the STEP trial. Nat Med. 2011;17(3):366-371.

27. Plotkin SA, Gilbert PB. Nomenclature for immune correlates of protection after vaccination. Clin Infect Dis. 2012;54(11):1615-1617.

28. Plotkin SA. Complex correlates of protection after vaccination. Clin Infect Dis. 2013;56(10):1458-1465.

29. Forthal DN, Gilbert PB, Landucci G, Phan T. Recombinant gp120 vaccine-induced antibodies inhibit clinical strains of HIV-1 in the presence of Fc receptor-bearing effector cells and correlate inversely with HIV infection rate. J Immunol. 2007;178(10):6596-6603.

30. Xiao P, et al. Multiple vaccine-elicited nonneutralizing antienvelope antibody activities contribute to protective efficacy by reducing both acute and chronic viremia following simian/human immunodeficiency virus SHIV89.6P challenge in rhesus macaques. J Virol. 2010;84(14):7161-7173.

31. Gómez-Román VR, et al. Vaccine-elicited antibodies mediate antibody-dependent cellular cytotoxicity correlated with significantly reduced acute viremia in rhesus macaques challenged with SIVmac251. J Immunol. 2005;174(4):2185-2189.

32. Hessell AJ, et al. Reduced cell-associated DNA and improved viral control in macaques following passive transfer of a single anti-V2 monoclonal antibody and repeated simian/human immunodeficiency virus challenges. J Virol. 2018;92(11):e02198-17.

33. Santra S, et al. Human non-neutralizing HIV-1 envelope monoclonal antibodies limit the number of founder viruses during SHIV mucosal infection in rhesus macaques. PLoS Pathog. 2015;11(8):e1005042.

34. Ackerman ME, et al. Enhanced phagocytic activity of HIV-specific antibodies correlates with natural production of immunoglobulins with skewed affinity for Fc $\gamma$ R2a and Fc $\gamma$ R2b. J Virol. 2013;87(10):5468-5476.

35. Bekker LG, et al. Subtype C ALVAC-HIV and bivalent subtype C gp120/MF59 HIV-1 vaccine in low-risk, HIV-uninfected, South African adults: a phase 1/2 trial. Lancet HIV. 2018;5(7):e366-e378.

36. Boesch AW, et al. Highly parallel characterization of IgG Fc binding interactions. MAbs. 2014;6(4):915-927.

37. Janda A, Bowen A, Greenspan NS, Casadevall A. Ig constant region effects on variable region structure and function. Front Microbiol. 2016;7:22.

38. Sela-Culang I, Alon S, Ofran Y. A systematic comparison of free and bound antibodies reveals binding-related conformational changes. J Immunol. 2012;189(10):4890-4899.

39. Bournazos S, Woof JM, Hart SP, Dransfield I. Functional and clinical consequences of $\mathrm{Fc}$ receptor polymorphic and copy number variants. Clin Exp Immunol. 2009;157(2):244-254.

40. Brown EP, et al. Multiplexed Fc array for evaluation of antigen-specific antibody effector profiles. J Immunol Methods. 2017;443:33-44.

41. Ackerman ME, et al. A robust, high-throughput assay to determine the phagocytic activity of clinical antibody samples. J Immunol Methods. 2011;366(1-2):8-19.

42. Wills S, et al. HIV-1-specific IgA monoclonal antibodies from an HIV-1 vaccinee mediate galactosylceramide blocking and phagocytosis. J Virol. 2018;92(7):e01552-17.

43. Dennison SM, et al. Vaccine-induced HIV-1 envelope gp120 constant region 1-specific antibodies expose a CD4-inducible epitope and block the interaction of HIV-1 gp140 with galactosylceramide. J Virol. 2014;88(16):9406-9417.

44. Watkins JD, et al. Anti-HIV IgA isotypes: differential virion capture and inhibition of transcytosis are linked to prevention of mucosal R5 SHIV transmission. AIDS. 2013;27(9):F13-F2O.

45. Sholukh AM, et al. Defense-in-depth by mucosally administered anti-HIV dimeric IgA2 and systemic IgG1 mAbs: complete protection of rhesus monkeys from mucosal SHIV challenge. Vaccine. 2015;33(17):2086-2095.

46. Freel SA, et al. Phenotypic and functional profile of HIV-inhibitory CD8 $\mathrm{T}$ cells elicited by natural infection and heterologous prime/boost vaccination. J Virol. 2010;84(10):4998-5006.

47. Yates NL, et al. Vaccine-induced Env V1-V2 IgG3 correlates with lower HIV-1 infection risk and declines soon after vaccination. Sci Trans Med. 2014;19;6(228):228ra39.

48. Li SS, et al. FCGR2C polymorphisms associate with HIV-1 vaccine protection in RV144 trial. JClin Invest. 2014;124(9):3879-3890.

49. Li SS, et al. Fc gamma receptor polymorphisms modulated the vaccine effect on HIV-1 risk in the HVTN 505 HIV vaccine trial [published online ahead of print August 21, 2019]. JVirol. https:// doi.org/10.1128/JVI.02041-18.

50. Yates NL, et al. HIV-1 envelope glycoproteins from diverse clades differentiate antibody responses and durability among vaccinees. JVirol. 2018;92(8):e01843-17.

51. deCamp A, et al. Global panel of HIV-1 Env reference strains for standardized assessments of vaccine-elicited neutralizing antibodies. J Virol. 2014;88(5):2489-2507.

52. Rolland M, et al. Increased HIV-1 vaccine efficacy against viruses with genetic signatures in Env V2. Nature. 2012;490(7420):417-420.

53. Horwitz JA, et al. Non-neutralizing antibodies alter the course of HIV-1 infection in vivo. Cell. 2017;170(4):637-648.e10.

54. Pollara J, et al. Application of area scaling analysis to identify natural killer cell and monocyte involvement in the GranToxiLux antibody dependent cell-mediated cytotoxicity assay. Cytometry A. 2018;93(4):436-447.

55. Richardson SI, et al. HIV-specific Fc effector function early in infection predicts the development of broadly neutralizing antibodies. PLOS Pathog. 2018;14(4):e1006987.

56. Bonsignori $\mathrm{M}$, et al. Two distinct broadly neutralizing antibody specificities of different clonal lineages in a single HIV-1-infected donor: implications for vaccine design. J Virol. 2012;86(8):4688-4692.

57 . Whittle JR, et al. Broadly neutralizing human antibody that recognizes the receptor-binding pocket of influenza virus hemagglutinin. Proc Natl Acad Sci U S A. 2011;108(34):14216-14221.

58. Tomaras GD, et al. Initial B-cell responses to transmitted human immunodeficiency virus type 1: virion-binding immunoglobulin $\mathrm{M}$ (IgM) and IgG antibodies followed by plasma anti-gp 41 antibodies with ineffective control of initial viremia. JVirol. 2008;82(24):12449-12463.

59. Seaton KE, et al. Computational analysis of antibody dynamics identifies recent HIV-1 infection. JCI Insight. 2017;2(24):94355.

60. Brown EP, et al. Optimization and qualification of an Fc array assay for assessments of antibodies against HIV-1/SIV. J Immunol Methods. 2018;455:24-33.

61. Liao HX, et al. Vaccine induction of antibodies against a structurally heterogeneous site of immune pressure within HIV-1 envelope protein variable regions 1 and 2. Immunity. 2013;38(1):176-186.

62. Breslow NE, Holubkov R. Weighted likelihood, pseudo-likelihood and maximum likelihood methods for logistic regression analysis of twostage data. Stat Med.1997;16(1-3):103-116.

63. Benjamini Y, Hochberg Y. Controlling the false discovery rate: a practical and powerful approach to multiple testing. JR Stat Soc Series B Stat Methodol. 1995;57(1):289-300.

64. Vansteelandt S, Goetghebeur E, Kenward MG, Molenberghs G. Ignorance and uncertainty regions as inferential tools in a sensitivity analysis. Statistica Sinica. 2006;16(3):953-979. 